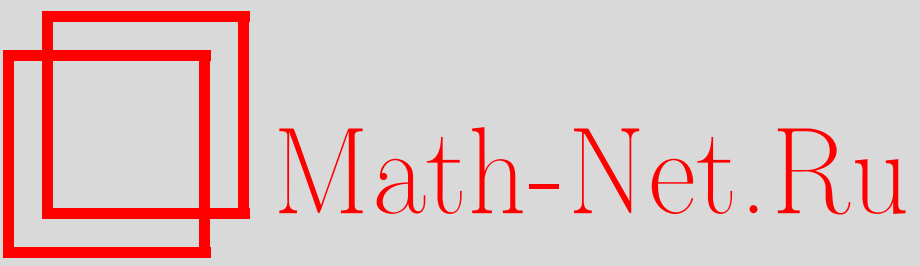

А. Н. Тимашев, Об одной аддитивной задаче теории чисел со случайным числом слагаемых, Матем. вопр. криптогр., 2013, том 4, выпуск 1, 129-150

DOI: https://doi.org/10.4213/mvk77

Использование Общероссийского математического портала Math-Net.Ru подразумевает, что вы прочитали и согласны с пользовательским соглашением

http://www.mathnet.ru/rus/agreement

Параметры загрузки:

IP: 52.23 .180 .231

26 апреля 2023 г., 12:32:02 
УДК $511.34+519.212 .2$

\title{
Об одной аддитивной задаче теории чисел со случайным числом слагаемых
}

\author{
А. Н. Тимашев \\ ООО «Центр сертификационных исследований», Москва
}

Получено 23.VI.2012

Получены асимптотические формулы для первых двух моментов числа решений уравнения $x_{1}^{m}+\ldots+x_{s}^{m}=N$ в целых неотрицательных числах, где $m, s, N$ - целые положительные числа, $m$ фиксировано, а $s$ - случайная величина. Рассмотрены случаи, когда распределение $s-1$ является биномиальным или пуассоновским. Доказательства основаны на методе перевала. Аналогичные вопросы рассматриваются и для числа целых неотрицательных решений неравенства $x_{1}^{m}+\ldots+x_{s}^{m} \leq N$.

Ключевые слова: диофантовы уравнения, производящие функции, метод перевала, первые моменты числа решений

On an additive problem of number theory with random number of summands

\section{A. N. Timashev}

\section{LLC «Certification Research Center», Moscow}

\begin{abstract}
Asymptotic formulas for the mean and variance of the number of nonnegative integer solutions of the equation $x_{1}^{m}+\ldots+x_{s}^{m}=N$ are obtained; here $m, s, N$ are integer positive numbers, $m=$ const, and $s$ is a random variable. Cases of the binomial and Poisson distributions of $s-1$ are considered. Proofs are based on the saddle point method. Analogous results for the number of nonnegative integer solutions of the inequality $x_{1}^{m}+\ldots+x_{s}^{m} \leq N$ are obtained also.
\end{abstract}

Key words: Diophantine equations, generating functions, saddle-point method, first moments of the number of solutions

Citation: Mathematical Aspects of Cryptography, 2013, vol. 4, no. 1, pp. 129-150 (Russian). 
Рассматривается уравнение

$$
x_{1}^{m}+\ldots+x_{s}^{m}=N
$$

в котором $m, s, N \in \mathbb{N}$, в целых неотрицательных числах $x_{1}, \ldots, x_{s}$. Всюду далее считаем, что $m=$ const. При $|z|<1$ положим

$$
f_{m}(z)=1+\sum_{n=1}^{\infty} z^{n^{m}} .
$$

Если $r_{k}(N)$ - число решений уравнения (1) в целых неотрицательных числах $x_{1}, \ldots, x_{k+1}$, где $s=k+1$, то согласно (2) и интегральной формуле Коши

$$
r_{k}(N)=\frac{1}{2 \pi i} \oint \frac{\left(f_{m}(z)\right)^{k+1}}{z^{N+1}} d z, \cdots k=0,1, \ldots,
$$

где интегрирование осуществляется по окружности $|z|=z_{0}$ радиуса $z_{0} \in(0,1)$, пробегаемой в положительном направлении.

Заметим, что вопрос о разрешимости уравнения (1) в целых положительных числах $x_{1}, \ldots, x_{s}$ при $m \geq 3$ называется проблемой Варинга [1; c. 177]. Асимптотическая формула для числа решений $t_{s}(N)$ этого уравнения при $N \rightarrow \infty$ и $s \geq \mathrm{cm}^{2} \ln m$ ( $c=$ const $\left.>0\right)$ найдена И. М. Виноградовым [1; c. 192]. Эта формула имеет вид

$$
t_{s}(N)=\frac{\left(\Gamma\left(1+\frac{1}{m}\right)\right)^{s}}{\Gamma\left(\frac{s}{m}\right)} \cdot \delta(N) \cdot N^{\frac{s}{m}-1}+O\left(N^{\frac{s}{m}-1-\frac{c_{0}}{m^{2}}}\right), c_{0}=\text { const }>0,
$$

где $\delta(N)$ - некоторый «сингулярный» ряд, сумма которого превосходит положительную постоянную. При этом предполагалось, что числа $s, m$ фиксированы.

Для случая, когда $m=$ const $\in \mathbb{N} u N, s \rightarrow \infty$ так, что $N s^{-1} \geq$ const $>1$, асимптотическая формула для величины $t_{s}(N)$ получена $Г$. А. Фрейманом [2] (см. также [3, с. 124]). В монографии автора [4, гл. IV] получены, в частности, асимптотические оценки величин $t_{s}(N)$, справедливые при $N, s \rightarrow \infty$ и фиксированном $m \in \mathbb{N}$ (рассмотрены три области изменения параметров $N, s)$. Там же выведена асимптотическая оценка величины $t_{s}(N)$ при условии, что $N, s \rightarrow \infty$ так, что $s^{m+1}=o(N)$. Эта оценка аналогична формуле 
И. М. Виноградова и формально получается из неё (за исключением вида остаточных членов), если считать, что $\delta(N)=1$.

Аналогичные оценки выведены и для числа решений неравенства

$$
x_{1}^{m}+\ldots+x_{s}^{m} \leq N
$$

в целых положительных числах $x_{1}, \ldots, x_{s}$.

Основным методом получения изложенных результатов в [4] является метод перевала в сочетании с теорией обобщенных схем размещения, ряд результатов которой изложен в [5] и [6].

В данной работе изучается случай, когда в уравнении (1) $x_{1}, \ldots, x_{s}$ целые неотрицательные числа. При этом предполагается, что число слагаемых s такая случайная величина, что

$$
\mathbf{P}\{s=k+1\}=p_{k}, k=0,1, \ldots, \quad \sum_{k=0}^{\infty} p_{k}=1 .
$$

Пусть $R \geq 1$ - радиус сходимости ряда

$$
P(z)=\sum_{k=0}^{\infty} p_{k} z^{k},
$$

тогда равенство (6) справедливо при $|z|<R$. Далее будем считать, что $R=+\infty$ (так будет, например, если случайная величина $s-1$ распределена по закону Пуассона с произвольным параметром $\lambda>0$ или имеет биномиальное распределение).

Пусть $\xi_{m}-$ случайная величина, равная числу решений уравнения (1) в целых неотрицательных числах $x_{1}, \ldots, x_{s}$ со случайным числом слагаемых, имеющим распределение вида (5). Тогда при $R=+\infty$ согласно (3), (6)

$$
\mathbf{E} \xi_{m}=\sum_{k=0}^{\infty} p_{k} r_{k}(N)=\frac{1}{2 \pi i} \oint_{0<|z|=z_{0}<1} \frac{f_{m}(z)}{z^{N+1}} P\left(f_{m}(z)\right) d z,
$$

функция $f_{m}(z)$ определена в (2).

Если $s-1$ имеет распределение Пуассона с параметром $\lambda$

$$
p_{k}=\frac{\lambda^{k}}{k !} e^{-\lambda}, k=0,1, \ldots, \lambda=\mathrm{const}>0,
$$


то при $z \in \mathbb{C}$

$$
P(z)=\exp (\lambda(z-1))
$$

так что из (7) следует равенство

$$
\mathbf{E} \xi_{m}=\frac{1}{2 \pi i} \oint_{0<|z|=z_{0}<1} \frac{f_{m}(z)}{z} \exp \left(\lambda\left(f_{m}(z)-1-\beta \ln z\right)\right) d z,
$$

где $\beta=N \lambda^{-1}$.

Пусть $N, \lambda \rightarrow \infty$ так, что

$$
0<\beta_{0} \leq \beta=N \lambda^{-1} \leq \beta_{1}<+\infty, \quad \beta_{0}, \beta_{1}=\text { const },
$$

тогда согласно (8), (9)

$$
\mathbf{E} \xi_{m}=\frac{1}{2 \pi i} \oint_{0<|z|=z_{0}<1} \frac{f_{m}(z)}{z} \exp (\lambda \omega(z)) d z
$$

где

$$
\omega(z)=f_{m}(z)-1-\beta \cdot \ln z
$$

Теорема 1. Пусть $s-1$ имеет распределение Пуассона спараметром $\lambda$ и $N, \lambda \rightarrow \infty$ так, что выполнены условия (9). Тогда равномерно относительно $\beta \in\left[\beta_{0}, \beta_{1}\right]$ прилюбом $m=1,2, \ldots$

$$
\mathbf{E} \xi_{m}=\frac{f_{m}\left(z_{0}\right)}{z_{0}^{N} \sqrt{2 \pi \lambda\left(z_{0}^{2} f_{m}^{\prime \prime}\left(z_{0}\right)+\beta\right)}} \exp \left(\lambda\left(f_{m}\left(z_{0}\right)-1\right)\right)\left(1+O\left(\lambda^{-1}\right)\right)
$$

где $z=z_{0}$ - единственный принадлежащий интервалу $(0,1)$ корень уравнения

$$
z f_{m}^{\prime}(z)=\sum_{n=1}^{\infty} n^{m} z^{n^{m}}=\beta
$$

величина $f_{m}\left(z_{0}\right)$ определяется из (2) при $z=z_{0}$ и согласно (2)

$$
z_{0}^{2} f_{m}^{\prime \prime}\left(z_{0}\right)=\sum_{n=2}^{\infty} n^{m}\left(n^{m}-1\right) z_{0}^{n^{m}}
$$


Замечание 1. При $m=1$ из (2), (13) нетрудно вывести, что при $\beta>0$

$$
z_{0}=1-\frac{\sqrt{1+4 \beta}-1}{2 \beta} \in(0,1),
$$

так что согласно (12)

$$
\mathbf{E} \xi_{1}=\frac{(1+4 \beta)^{1 / 4}+(1+4 \beta)^{-1 / 4}}{2 \sqrt{2 \pi N}} z_{0}^{-N} \exp \left(\frac{\lambda}{2}(\sqrt{1+4 \beta}-1)\right)\left(1+O\left(\lambda^{-1}\right)\right) .
$$

Пусть $\eta_{m}-$ случайная величина, равная числу решений неравенства

$$
x_{1}^{m}+\ldots+x_{s}^{m} \leq N
$$

в целых неотрицательных числах $x_{1}, \ldots, x_{s}$ при условии, что $s$ - случайная величина, имеющая распределение вида (5), для которого $R=+\infty$.

Теорема 2. Пусть $s-1$ имеет распределение Пуассона с параметром $\lambda u N, \lambda \rightarrow \infty$ так, что $\lambda=o\left(N^{1 / 3}\right)$. Тогда

$$
\begin{gathered}
\mathbf{E} \xi_{1}=\frac{1}{2 \sqrt{\pi}}(\lambda N)^{-1 / 4} \exp \left(2 \sqrt{\lambda N}-\frac{\lambda}{2}\right)(1+o(1)), \\
\mathbf{E} \eta_{1}=\sqrt{\frac{N}{\lambda}} \mathbf{E} \xi_{1}(1+o(1)) .
\end{gathered}
$$

При фиксированном значении $s=k+1$ число решений неравенства (14) равно

$$
T_{k}(N)=\sum_{l=0}^{N} r_{k}(l), \quad k=0,1, \ldots ;
$$

величина $r_{k}(l)$ определена в (3) с заменой $N$ на $l$. Поэтому согласно (3)

$$
\begin{gathered}
T_{k}(N)=\frac{1}{2 \pi i} \oint_{0<|z|=z_{0}<1} \frac{\left(f_{m}(z)\right)^{k+1}}{z} \sum_{l=0}^{N} z^{-l} d z= \\
=\frac{1}{2 \pi i} \oint_{0<|z|=z_{0}<1} \frac{\left(f_{m}(z)\right)^{k+1}}{z^{N+1}(1-z)} d z-\frac{1}{2 \pi i} \oint_{0<|z|=z_{0}<1} \frac{\left(f_{m}(z)\right)^{k+1}}{1-z} d z,
\end{gathered}
$$

причем второй интеграл согласно интегральной теореме Коши равен нулю.

Таким образом,

$$
\mathbf{E} \eta_{m}=\sum_{k=0}^{\infty} p_{k} T_{k}(N)=\frac{1}{2 \pi i} \oint_{0<|z|=z_{0}<1} \frac{f_{m}(z)}{z^{N+1}(1-z)} P\left(f_{m}(z)\right) d z .
$$


Следствие 1. При условиях теоремы 1 равномерно относительно $\beta \in\left[\beta_{0}, \beta_{1}\right]$

$$
\mathbf{E} \eta_{m}=\left(1-z_{0}\right)^{-1} \mathbf{E} \xi_{m}\left(1+O\left(\lambda^{-1}\right)\right), \quad m=1,2, \ldots
$$

В частности, при $m=1$

$$
\mathbf{E} \eta_{1}=\frac{\sqrt{1+4 \beta}+1}{2} \mathbf{E} \xi_{1}\left(1+O\left(\lambda^{-1}\right)\right) .
$$

Теорема 3. Пусть $s-1$ имеет распределение Пуассона с параметром $\lambda u N, \lambda \rightarrow \infty$ так, что $\lambda=o\left(N^{1 / 4}\right)$. Тогда при $m=2$

$$
\begin{gathered}
\mathbf{E} \xi_{2}=\frac{1}{2^{\frac{1}{6}} \sqrt{3 N}} \exp \left(\frac{3}{2} \sqrt[3]{\frac{\pi N \lambda^{2}}{2}}-\frac{\lambda}{2}\right)(1+o(1)), \\
\mathbf{E} \eta_{2}=2 \sqrt[3]{\frac{2 N^{2}}{\pi \lambda^{2}}} \mathbf{E} \xi_{2}(1+o(1)) .
\end{gathered}
$$

Теорема 4. Пусть $s-1$ имеет распределение Пуассона с параметром $\lambda u N, \lambda \rightarrow \infty$ так, что

$$
N=o\left(\lambda^{1-2^{-m}}\right)
$$

Тогда

$$
\begin{aligned}
\mathbf{E} \xi_{m} & =\frac{1}{\sqrt{2 \pi N}}\left(\frac{\lambda e}{N}\right)^{N}(1+o(1)), \\
\mathbf{E} \eta_{m} & =\mathbf{E} \xi_{m}(1+o(1)), \quad m=1,2, \ldots
\end{aligned}
$$

Замечание 2. При $m=1$ утверждение теоремы 4 можно уточнить. Можно показать, что если $N, \lambda \rightarrow \infty$ так, что $N=o\left(\lambda^{2 / 3}\right)$, то

$$
\mathbf{E} \xi_{1}=\frac{1}{\sqrt{2 \pi N}}\left(\frac{\lambda}{N}\right)^{N} \exp \left(\frac{N^{2}}{\lambda}+N\right)(1+o(1)) .
$$

Предположим теперь, что $n \in \mathbb{N}, p \in(0,1)$ и $s-1$ имеет биномиальное распределение с параметрами $n$ и $p$. Далее считаем, что $p=$ const. При таких условиях

$$
p_{k}=\left(\begin{array}{l}
n \\
k
\end{array}\right) p^{k}(1-p)^{n-k}, \quad k=0,1, \ldots, n,
$$


так, что

$$
P(z)=(1+p(z-1))^{n}
$$

и согласно (7) (с заменой $\mathrm{z}_{0}$ на $\mathrm{z}_{1}$ )

$$
\begin{gathered}
\mathbf{E} \xi_{m}=\frac{1}{2 \pi i} \oint_{0<|z|=z_{1}<1} \frac{f_{m}(z)}{z^{N+1}}\left(1+p\left(f_{m}(z)-1\right)\right)^{n} d z= \\
=\frac{1}{2 \pi i} \oint_{0<|z|=z_{1}<1} \frac{f_{m}(z)}{z} \exp (N \omega(z)) d z,
\end{gathered}
$$

где

$$
\omega(z)=\beta \ln \left(1+p\left(f_{m}(z)-1\right)\right)-\ln z, \quad \beta=n N^{-1},
$$

величина $\beta$ и функция $\omega(z)$ здесь имеют иной смысл, нежели в (9), (11).

Теорема 5. Пусть $s-1$ имеет биномиальное распределение с параметрами $n, p$ и $n, N \rightarrow \infty$ так, что

$$
0<\beta_{0} \leq \beta=n N^{-1} \leq \beta_{1}<+\infty, \beta_{0}, \beta_{1}=\text { const. }
$$

Тогда равномерно относительно $\beta \in\left[\beta_{0}, \beta_{1}\right]$

$$
\begin{aligned}
\mathbf{E} \xi_{m}= & \frac{1}{\sqrt{2 \pi N\left(z_{1} f_{m}^{\prime \prime}\left(z_{1}\right)\left(f_{m}^{\prime}\left(z_{1}\right)\right)^{-1}+1-\beta^{-1}\right)}} z_{1}^{-N} f_{m}\left(z_{1}\right) \times \\
& \times\left(1+p\left(f_{m}\left(z_{1}\right)-1\right)\right)^{n}\left(1+O\left(N^{-1}\right)\right), \quad m=1,2, \ldots,
\end{aligned}
$$

где $z_{1}$ - единственный принадлежащчий интервалу $(0,1)$ корень уравнения

$$
\beta z f_{m}^{\prime}(z)-f_{m}(z)=p^{-1}-1 \text {. }
$$

Замечание 3. При $\beta \rightarrow+\infty$

$$
z_{1}=z_{1}(\beta) \rightarrow 0 .
$$

Следствие 2. При условиях теоремы 5

$$
\mathbf{E} \eta_{m}=\left(1-z_{1}\right)^{-1} \mathbf{E} \xi_{m}\left(1+O\left(N^{-1}\right)\right), m=1,2, \ldots
$$

Следствие 3. При условиях теоремь 5 и $\mathrm{m}=1$

$$
\mathbf{E} \xi_{1}=\sqrt{\frac{\beta}{2 \pi N\left(1-z_{1}\right)\left((1+\beta) z_{1}+\beta-1\right)}} z_{1}^{-N}\left(1+\frac{p z_{1}}{1-z_{1}}\right)^{n}\left(1+O\left(N^{-1}\right)\right),
$$


где

$$
\begin{gathered}
z_{1}=\frac{2-(1-\beta) p-\sqrt{(1-\beta)^{2} p^{2}+4 \beta p}}{2(1-p)} \in(0,1), \\
\mathbf{E} \eta_{1}=\frac{2(1-p)}{\sqrt{(1-\beta)^{2} p^{2}+4 \beta p}-(1+\beta) p} \mathbf{E} \xi_{1}\left(1+O\left(N^{-1}\right)\right) .
\end{gathered}
$$

Теорема 6. Пусть $s-1$ имеет биномиальное распределение с параметрами $n, p$ и $n, N \rightarrow \infty$ так, что $N=o(\sqrt{n})$. Тогда

$$
\begin{gathered}
\mathbf{E} \xi_{m}=\frac{1}{\sqrt{2 \pi N}}\left(\frac{n p e}{N}\right)^{N}(1+o(1)), \\
\mathbf{E} \eta_{m}=\mathbf{E} \xi_{m}(1+o(1)), \quad m=1,2, \ldots .
\end{gathered}
$$

Теорема 7. Пусть $s-1$ имеет биномиальное распределение с параметрами $n, p$ и $n, N \rightarrow \infty$ так, что $n=o\left(N^{2 / 3}\right)$. Тогда при $m=1$

$$
\begin{gathered}
\mathbf{E} \xi_{1}=\frac{1}{\sqrt{2 \pi n}} \exp \left(\left(\frac{1}{p}-\frac{1}{2}\right) \frac{n^{2}}{N}+n\right)\left(\frac{p N}{n}\right)^{n}(1+o(1)), \\
\mathbf{E} \eta_{1}=\frac{N}{n} \mathbf{E} \xi_{1}(1+o(1)) .
\end{gathered}
$$

Теорема 8. Пусть $s-1$ имеет биномиальное распределение с параметрами $n, p$ и $n, N \rightarrow \infty$ так, что $n=o\left(N^{1 / 3}\right)$. Тогда

$$
\begin{gathered}
\mathbf{E} \xi_{2}=\frac{p^{n}}{2 \sqrt{2 N}}\left(\frac{\pi e N}{2 n}\right)^{\frac{n}{2}}(1+o(1)), \\
\mathbf{E} \eta_{2}=\frac{2 N}{n} \mathbf{E} \xi_{2}(1+o(1)) .
\end{gathered}
$$

Для оценки дисперсии $\mathbf{D} \xi_{m}$ следует найти асимптотику второго момента $\mathbf{E} \xi_{m}^{2}$. Во избежание громоздких выкладок ограничимся случаем $m=1$. В этом случае уравнение (1) при фиксированном значении $s \in \mathbb{N}$ имеет $\left(\begin{array}{c}N+s-1 \\ s-1\end{array}\right)$ решений. Если $s-$ случайная величина, имеющая распределение вида (5), то

$$
\mathbf{E} \xi_{1}^{2}=\sum_{k=0}^{\infty} p_{k}\left(C_{N+k}^{k}\right)^{2}
$$


Для сходимости ряда (23) достаточно, чтобы $R>1$. Известно [7, с. 73], что

$$
\left(C_{N+k}^{k}\right)^{2}=\frac{1}{(2 \pi i)^{2}} \oint_{|z|=z^{*}>0} \oint_{|u|=u^{*}>0} \frac{(1+z)^{N+k}(1+u z)^{N+k}}{z^{N+k+1} u^{k+1}} d z d u, \quad N, k=0,1, \ldots
$$

Поэтому согласно (23) при $R>1$

$$
\mathbf{E} \xi_{1}^{2}=\frac{1}{(2 \pi i)^{2}} \oint_{|z|=z^{*}>0|u|=u^{*}>0} \frac{\left(1+z^{-1}\right)^{N}(1+u z)^{N}}{z u} P\left(1+z+\frac{1}{u}+\frac{1}{u z}\right) d z d u,(24)
$$

где

$$
\left|\frac{(1+z)(1+u z)}{z u}\right|<R
$$

или, что то же самое,

$$
|(1+z)(1+u z)|<R|z u|=R z^{*} u^{*}
$$

для чего, в свою очередь, достаточно, чтобы при $R>1$

$$
0<z^{*}<R-1 \quad \text { и } \quad u^{*}>\frac{1+z^{*}}{z^{*}\left(R-1-z^{*}\right)} .
$$

В случае, когда $R=+\infty$, в соотношении (24) можно интегрировать по произвольным окружностям $|z|=z^{*}>0 ;|u|=u^{*}>0$, пробегаемым в положительном направлении. Это соотношение можно представить в следующем виде:

$$
\mathbf{E} \xi_{1}^{2}=\frac{1}{(2 \pi i)^{2}} \oint_{|z|=z^{*}>0} \oint_{|u|=u^{*}>0} \frac{\left(u+u z+u^{2} z+u^{2} z^{2}\right)^{N}}{z^{N+1} u^{N+1}} P\left(\left(1+z^{-1}\right)\left(z+u^{-1}\right)\right) d z d u .
$$

Теорема 9. При условиях теоремы 1 и $m=1$ равномерно относительно $\beta \in\left[\beta_{0}, \beta_{1}\right]$

$$
\mathbf{E} \xi_{1}^{2}=\frac{\left(1+\beta^{-1 / 4}\right)^{2 N}}{2 \pi N \sqrt{\Delta}} \exp \left(\lambda\left(\sqrt{\beta}+2 \beta^{1 / 4}\right)\right)\left(1+O\left(N^{-1}\right)\right)
$$

где

$$
\Delta=\sqrt{\beta} \cdot\left(1+\beta^{1 / 4}\right)^{-4}+\frac{2}{\sqrt{\beta}}\left(1+\beta^{1 / 4}\right)^{-1}+\beta^{-3 / 2}\left(1+2 \beta^{1 / 4}\right)>0 .
$$


Теорема 10. При условиях теоремы 5 и $m=1$ равномерно относительно $\beta \in\left[\beta_{0}, \beta_{1}\right]$

$$
\mathbf{E} \xi_{1}^{2}=\frac{1}{2 \pi N \sqrt{\Delta}}\left(1+\frac{1}{z^{*}}\right)^{2 N}\left(1+p z^{*}\left(z^{*}+2\right)\right)^{n}\left(1+O\left(N^{-1}\right)\right),
$$

где $z^{*}-$ единственный положительный корень уравнения

$$
\begin{aligned}
& \beta z^{3}+(2 \beta-1) z^{2}+(\beta-2) z-p^{-1}=0, \\
& \Delta=(a+b)\left(a+b+\frac{2(1-p) b^{2}}{\beta p}\right)>0, \\
& a=z^{*}\left(1+z^{*}\right)^{-2}, \quad b=\frac{\beta p z^{*}}{1+p z^{*}\left(z^{*}+2\right)} .
\end{aligned}
$$

Замечание 4. Оценки (26), (27) имеют довольно высокую точность. Так, например, если положить $p=1 / 2$ в теореме 10 , то из точной формулы

$$
\mathbf{E} \xi_{1}^{2}=2^{-n} \sum_{k=0}^{n} C_{n}^{k} \prod_{j=1}^{k}\left(1+\frac{N}{j}\right)^{2}
$$

при $n=10, N=1000, \beta=n N^{-1}=0.01$ следует, что $\mathbf{E} \xi_{1}^{2}=8.2833 \ldots \cdot 10^{43}$. При таких же условиях оценка (27) дает значение $8.4225 \ldots \cdot 10^{43}$ с относительной погрешностью порядка $0,0168 \ldots$.

Доказательство теоремы 1. Если справедливо соотношение (13), то согласно (11)

$$
\omega^{\prime}(z)=f_{m}^{\prime}(z)-\beta z^{-1}=0 .
$$

Если $q_{m}(z)=z f_{m}^{\prime}(z)$ при $|z|<1$, то $q_{m}(0)=0 ; \lim _{z \rightarrow 1} q_{m}(z)=+\infty$ и функция $q_{m}(z)$ строго возрастает и непрерывна на полуинтервале $[0,1)$, поскольку при $z \in[0,1)$

$$
q_{m}^{\prime}(z)=\sum_{n=1}^{\infty} n^{2 m} z^{n^{m}-1}>0 .
$$

Таким образом, уравнение (13) при любом $\beta>0$ имеет единственный корень $z=z_{0} \in(0,1)$.

Далее получаем

$$
\omega^{\prime \prime}\left(z_{0}\right)=f_{m}^{\prime \prime}\left(z_{0}\right)+\frac{\beta}{z_{0}^{2}}>0,
$$

и если $|z|=z_{0}$ и $z \neq z_{0}$, то $\operatorname{Re} \omega\left(z_{0}\right)>\operatorname{Re} \omega(z)$. 
Следовательно, на окружности $|z|=z_{0}$ точка $z=z_{0}$ имеет наибольшую высоту, причем эта точка является точкой перевала первого порядка функции $\omega$. Используя (8)-(10) и применяя стандартную технику метода перевала, из этих замечаний выводим оценку (12). Теорема 1 доказана.

Доказательство теоремы 2. Используем оценку (12), имея в виду, что при условиях теоремы 2 эта оценка остается справедливой с заменой остаточного члена $O\left(\lambda^{-1}\right)$ на $o(1)$. Если $\lambda=o\left(\mathrm{~N}^{1 / 3}\right)$, то при $\beta=N \lambda^{-1} \rightarrow \infty$ имеем $\lambda=o(\sqrt{\beta})$, так что

$$
\exp \left(\frac{\lambda}{2}(\sqrt{1+4 \beta}-1)\right)=\exp \left(\lambda \sqrt{\beta}-\frac{\lambda}{2}\right)(1+o(1))=\exp \left(\sqrt{\lambda N}-\frac{\lambda}{2}\right)(1+o(1)) .
$$

Кроме того, согласно (13) $\mathrm{z}_{0} \rightarrow 1-$ при $\beta \rightarrow \infty$, и с учетом замечания 1

$$
\ln z_{0}=-\frac{1}{\sqrt{\beta}}+O\left(\beta^{-3 / 2}\right)
$$

поэтому

$$
z_{0}^{-N}=\exp (\sqrt{\lambda N})(1+o(1))
$$

Подставляя эти оценки в асимптотическую формулу для $\mathbf{E} \xi_{1}$, выписанную в замечании 1, после элементарных преобразований получаем нужный результат. Поскольку при условиях теоремы 2

$$
\left(1-z_{0}\right)^{-1}=\sqrt{\beta}(1+o(1)),
$$

с учетом (15) при $m=1$ получаем оценку $\mathbf{E} \eta_{1}$. Теорема 2 доказана.

Доказательство теоремы 3. Положим при $|z|<1$

$$
g_{2}(z)=\frac{1}{2} \sqrt{\frac{\pi}{1-z}} .
$$

Известно [4, гл. IV], что

$$
\lim _{z \rightarrow 1}\left(f_{2}(z)-g_{2}(z)\right)=\frac{1}{2} .
$$

Пусть при условии $\operatorname{Re} t>0$

$$
\varphi(t)=1+2 \sum_{n=1}^{\infty} \exp \left(-\pi \cdot n^{2} t\right) .
$$


Если положить $z=\exp (-\pi t)$, то $|z|<1$ тогда и только тогда, когда $\operatorname{Re} t>0$. Кроме того,

$$
\varphi(t)=1+2 \sum_{n=1}^{\infty} z^{n^{2}}=2 f_{2}(z)-1 .
$$
функции

Известно также [8, с. 39], что согласно соотношению для тета-

$$
\begin{gathered}
\varphi\left(\frac{1}{t}\right)=\sqrt{t} \varphi(t) . \\
z=\exp (-\pi t) \text { следует, что } t=-\frac{\ln z}{\pi} . \text { С учетом } \\
f_{2}(z)=\frac{1}{2}\left(1-\sqrt{-\frac{\pi}{\ln z}}\right)+\sqrt{-\frac{\pi}{\ln z}} f_{2}\left(e^{\pi^{2} / \ln z}\right) .
\end{gathered}
$$

Из равенства $z=\exp (-\pi t)$ следует, что $t=-\frac{\ln z}{\pi}$. С учетом (28) получаем

При $z \rightarrow 1$

$$
-\frac{\pi}{\ln z}=\frac{\pi}{1-z}-\frac{\pi}{2}+O(1-z)
$$

так что

$$
\sqrt{-\frac{\pi}{\ln z}}=\sqrt{\frac{\pi}{1-z}}\left(1-\frac{1-z}{4}+O\left((1-z)^{2}\right)\right) .
$$

Подставляя эти оценки в (29), после элементарных преобразований приходим к равенству: при $z \rightarrow 1-$

$$
f_{2}(z)=\frac{1}{2} \sqrt{\frac{\pi}{1-z}}+\frac{1}{2}-\frac{1}{8} \cdot \sqrt{\pi(1-z)}+O\left((1-z)^{\frac{3}{2}}\right)
$$

(при этом остаточный член вида $O\left(e^{\pi^{2} / \ln z}\right)$ можно опустить).

Из уравнения (13) при $m=2$ следует, что при условиях теоремы 3

$$
z_{0} f_{2}^{\prime}\left(z_{0}\right)=\beta=N \lambda^{-1} \rightarrow \infty,
$$

так что $z_{0}=z_{0}(\beta) \rightarrow 1-$, и поэтому $f_{2}^{\prime}\left(z_{0}\right) \sim \beta$. В [4; гл. IV] показано, что при $z \rightarrow 1-$

$$
f_{2}^{\prime}(z) \sim g_{2}^{\prime}(z)=\frac{\sqrt{\pi}}{4}(1-z)^{-3 / 2}
$$


Следовательно,

$$
1-z_{0} \sim \frac{1}{2} \sqrt[3]{\frac{\pi}{2}} \beta^{-2 / 3}
$$

Таким образом,

$$
N\left(1-z_{0}\right)^{2}=O\left(N \beta^{-4 / 3}\right)=o(1)
$$

если $\lambda=o\left(N^{1 / 4}\right)$. Отсюда выводим, что

$$
\begin{gathered}
\ln \left(z_{0}^{-N}\right)=-N \ln \left(1-\left(1-z_{0}\right)\right)=N\left(1-z_{0}\right)+O\left(N\left(1-z_{0}\right)^{2}\right)= \\
=N\left(1-z_{0}\right)+o(1),
\end{gathered}
$$

то есть

$$
z_{0}^{-N}=\exp \left(N\left(1-z_{0}\right)\right)(1+o(1))
$$

Далее получаем

$$
f_{2}^{\prime}\left(z_{0}\right)=\frac{\beta}{z_{0}}=\beta\left(2-z_{0}\right)+O\left(\beta\left(1-z_{0}\right)^{2}\right)=\beta+\frac{1}{2} \sqrt[3]{\frac{\pi}{2}} \beta^{1 / 3}+o\left(\beta^{1 / 3}\right) .
$$

Из результатов, изложенных в [4, гл. IV], следует, что при $z_{0}=e^{-\pi t_{0}} \rightarrow 1-$ (или, что эквивалентно, при $t_{0} \rightarrow 0+$ )

$$
\begin{gathered}
z_{0} f_{2}^{\prime}\left(z_{0}\right)=\frac{\varphi\left(t_{0}\right)}{4 \pi t_{0}}+O\left(t_{0}^{-5 / 2} e^{-\pi / t_{0}}\right)= \\
=\frac{\sqrt{\pi}}{4}\left(1-z_{0}\right)^{-3 / 2}-\frac{3 \sqrt{\pi}}{16}\left(1-z_{0}\right)^{-1 / 2}+O\left(\left(1-z_{0}\right)^{1 / 2}\right)=\beta ;
\end{gathered}
$$

при этом используются оценки

$$
\begin{gathered}
-\ln z_{0}=\left(1-z_{0}\right)\left(1+\frac{1}{2}\left(1-z_{0}\right)+O\left(\left(1-z_{0}\right)^{2}\right)\right), \\
t_{0}=-\frac{\ln z_{0}}{\pi} \sim \frac{1-z_{0}}{\pi} .
\end{gathered}
$$

Из полученных оценок нетрудно получить, что

$$
1-z_{0}=\frac{1}{2} \sqrt[3]{\frac{\pi}{2}} \cdot \beta^{-\frac{2}{3}}-\frac{1}{8} \cdot \sqrt[3]{\frac{\pi^{2}}{4}} \cdot \beta^{-\frac{4}{3}}+o\left(\beta^{-\frac{4}{3}}\right)
$$


то есть

$$
N\left(1-z_{0}\right)=\frac{1}{2} \sqrt[3]{\frac{\pi N \lambda^{2}}{2}}+O\left(N \beta^{-4 / 3}\right)=\frac{1}{2} \sqrt[3]{\frac{\pi N \lambda^{2}}{2}}+o(1)
$$

поскольку из условия $\lambda=o\left(N^{1 / 4}\right)$ следует, что $N \beta^{-4 / 3}=o(1)$.

С учетом (31) имеем

$$
z_{0}^{-N}=\exp \left(\frac{1}{2} \sqrt[3]{\frac{\pi N \lambda^{2}}{2}}\right)(1+o(1)) .
$$

В [4, гл. IV] показано также, что

$$
f_{2}^{\prime \prime}\left(z_{0}\right) \sim \frac{3 \sqrt{\pi}}{8}\left(1-z_{0}\right)^{-5 / 2}
$$

и так как

$$
1-z_{0} \sim \frac{1}{2} \sqrt[3]{\frac{\pi}{2}} \beta^{-2 / 3}
$$

To

$$
\left(1-z_{0}\right)^{-5 / 2} \sim C \beta^{5 / 3} \text {, где } C=2^{5 / 3} \cdot \pi^{-5 / 6} .
$$

Используя эти оценки, а также соотношение

$$
f_{2}\left(z_{0}\right) \sim \frac{1}{2} \sqrt{\frac{\pi}{1-z_{0}}}
$$

получаем, что

$$
\frac{f_{2}\left(z_{0}\right)}{\sqrt{2 \pi \lambda\left(z_{0}^{2} f_{2}^{\prime \prime}\left(z_{0}\right)+\beta\right)}} \sim \frac{1}{2^{1 / 6} \sqrt{3 N}} .
$$

Если $\lambda=o\left(N^{1 / 4}\right)$, то с учетом (30)

$$
\exp \left(\lambda\left(f_{2}\left(z_{0}\right)-1\right)\right)=\exp \left(\frac{\lambda}{2}\left(\sqrt{\frac{\pi}{1-z_{0}}}-1\right)\right)(1+o(1)),
$$

причем

$$
\frac{\lambda}{2} \sqrt{\frac{\pi}{1-z_{0}}}=\sqrt[3]{\frac{\pi N \lambda^{2}}{2}}+o(1)
$$


и поэтому

$$
\exp \left(\lambda\left(f_{2}\left(z_{0}\right)-1\right)\right)=\exp \left(\sqrt[3]{\frac{\pi N \lambda^{2}}{2}}-\frac{\lambda}{2}\right)(1+o(1))
$$

Так как при условиях теоремы 3 оценка (12) остается справедливой с заменой остаточного члена $O\left(\lambda^{-1}\right)$ на $o(1)$, то из соотношений (12) (при $m=2)$, (32)-(34) следует (16). Формула (17) следует из (15) (при $m=2$ ), а также из соотношения

$$
\left(1-z_{0}\right)^{-1} \sim 2 \sqrt[3]{\frac{2 \beta^{2}}{\pi}}
$$

Тем самым теорема 3 доказана.

Доказательство теоремы 4. При условиях теоремы $4 \beta=N \cdot \lambda^{-1} \rightarrow 0$, так что уравнение (13) имеет такой единственный корень $z=z_{0} \in(0,1)$, что

$$
z_{0} f_{m}^{\prime}\left(z_{0}\right)=\sum_{n=1}^{\infty} n^{m} z_{0}^{n^{m}}=z_{0}+O\left(z_{0}^{2^{m}}\right)=\beta,
$$

и поэтому

$$
z_{0}=\beta\left(1+O\left(\beta^{2^{m}-1}\right)\right)
$$

Следовательно,

$$
\lambda\left(f_{m}\left(z_{0}\right)-1\right)=\lambda \sum_{n=1}^{\infty} z_{0}^{n^{m}}=N+O\left(\lambda \beta^{2^{m}}\right)=N+o(1)
$$

если

$$
N=o\left(\lambda^{1-2^{-m}}\right)
$$

При таком условии

$$
\begin{gathered}
\exp \left(\lambda\left(f_{m}\left(z_{0}\right)-1\right)\right)=e^{N}(1+o(1)) \\
z_{0}^{-N}=\exp \left(-N \ln z_{0}\right)=\exp \left(-N \ln \beta+O\left(N \beta^{2^{m}-1}\right)\right)=\left(\frac{\lambda}{N}\right)^{N}(1+o(1)) .
\end{gathered}
$$

Далее имеем

$$
f_{m}\left(z_{0}\right)=1+o(1), \quad z_{0}^{2} f_{m}^{\prime \prime}\left(z_{0}\right) \sim 2^{m}\left(2^{m}-1\right) \beta^{2^{m}}=o(\beta),
$$


поскольку $2^{m} \geq 2$ при любом $m \in \mathbb{N}$. Поэтому

$$
\frac{f_{m}\left(z_{0}\right)}{\sqrt{2 \pi \lambda\left(z_{0}^{2} f_{m}^{\prime \prime}\left(z_{0}\right)+\beta\right)}}=\frac{1+o(1)}{\sqrt{2 \pi N}} .
$$

Из этих оценок и соотношения (12) получаем асимптотическую формулу для $\mathbf{E} \xi_{m}$, приведенную в формулировке теоремы 4. Так как $z_{0}=o(1)$, то $\left(1-z_{0}\right)^{-1}=1+o(1)$, и поэтому с учетом (15)

$$
\mathbf{E} \eta_{m}=\mathbf{E} \xi_{m}(1+o(1))
$$

Теорема 4 доказана.

Доказательство теоремы 5. Доказательство оценки (21) проводится аналогично обоснованию оценки (12) (вместо равенств (10), (11) следует воспользоваться соотношениями (18), (19) и учесть, что уравнение $\omega^{\prime}(z)=0$ имеет единственный корень $z=z_{1} \in(0,1)$ и равносильно уравнению (22)). Тем самым теорема 5 доказана.

Доказательство теоремы 6. Имеем $\beta=n N^{-1} \rightarrow \infty$, если $n, N \rightarrow \infty$ так, что $N=o(\sqrt{n})$. Согласно замечанию 3 отсюда следует, что $z_{1}=z_{1}(\beta)=o(1)$, и поэтому

$$
f_{m}\left(z_{1}\right)-1 \sim z_{1}
$$

так что

$$
\beta z_{1} f_{m}^{\prime}\left(z_{1}\right)=\beta\left(z_{1}+O\left(z_{1}^{2^{m}}\right)\right) \sim \beta z_{1}
$$

Согласно (22) имеем $\beta z_{1} \sim p^{-1}$, то есть

$$
z_{1}=\frac{1}{\beta p}(1+o(1)) \text {. }
$$

Если положить

$$
z_{1}=\frac{1}{\beta p}(1+\varepsilon(\beta))
$$

то $\varepsilon(\beta) \rightarrow 0$ при $\beta \rightarrow \infty$.

Далее,

$$
\beta z_{1} f_{m}^{\prime}\left(z_{1}\right)-f_{m}\left(z_{1}\right)+1=(\beta-1) z_{1}+\left(\beta 2^{m}-1\right) z_{1}^{2^{m}}+O\left(\beta^{-3^{m}+1}\right)=p^{-1},
$$


и отсюда нетрудно получить, что

$$
\varepsilon(\beta)=O\left(\beta^{-1}\right), \quad z_{1}=\frac{1}{\beta p}\left(1+O\left(\beta^{-1}\right)\right) .
$$

Таким образом,

$$
\begin{gathered}
n \ln \left(1+p\left(f_{m}\left(z_{1}\right)-1\right)\right)=n p\left(f_{m}\left(z_{1}\right)-1\right)+O\left(n \beta^{-2}\right)= \\
=n \beta^{-1}+O\left(n \beta^{-2}\right)=n \beta^{-1}+o(1),
\end{gathered}
$$

если $N=o(\sqrt{n})$. Значит,

$$
\left(1+p\left(f_{m}\left(z_{1}\right)-1\right)\right)^{n}=e^{N}(1+o(1)) .
$$

Кроме того,

$$
-N \ln z_{1}=N \ln (\beta p)+O\left(\frac{N^{2}}{n}\right)=N \ln (\beta p)+o(1),
$$

так что

$$
\begin{gathered}
z_{1}^{-N}=\left(\frac{n p}{N}\right)^{N}(1+o(1)), \\
z_{1} f_{m}^{\prime \prime}\left(z_{1}\right)\left(f_{m}^{\prime}\left(z_{1}\right)\right)^{-1} \sim \frac{2^{m}\left(2^{m}-1\right)}{(\beta p)^{2^{m}-1}}=o(1), \quad m=1,2, \ldots .
\end{gathered}
$$

С учетом этих оценок получаем асимптотическую формулу для $\mathbf{E} \boldsymbol{\xi}_{m}$, приведенную в формулировке теоремы 6. Так как $z_{1}=o(1)$, то $\mathbf{E} \eta_{m} \sim\left(1-z_{1}\right)^{-1} \mathbf{E} \xi_{m} \sim \mathbf{E} \xi_{m}$. Теорема 6 доказана.

Доказательство теоремы 7. При сформулированных условиях $\beta=n N^{-1}=o\left(N^{-1 / 3}\right) \rightarrow 0$, причем утверждение теоремы 5 остается справедливым с заменой в (21) остаточного члена $O\left(N^{-1}\right)$ на $o(1)$. При $m=1$ с учетом следствия 3 после элементарных преобразований получаем оценку $\mathbf{E} \xi_{1}$, приведенную в формулировке теоремы 7. Так как $\left(1-z_{1}\right)^{-1} \sim \beta^{-1}=\frac{N}{n}$, то $\mathbf{E} \eta_{1} \sim \frac{N}{n} \mathbf{E} \xi_{1}$. Теорема 7 доказана. 
Доказательство теоремы 8. Имеем $\beta=n N^{-1}=o\left(N^{-2 / 3}\right) \rightarrow 0$, причем при $m=2$ утверждение теоремы 5 также остается справедливым (с заменой в (21) остаточного члена $O\left(N^{-1}\right)$ на $o(1)$. Используя оценки, полученные при доказательстве теоремы 3 , и замечая, что $z_{1} \rightarrow 1-$, выводим соотношения:

$$
\begin{gathered}
f_{2}\left(z_{1}\right)=\frac{1}{2} \sqrt{\frac{\pi}{1-z_{1}}}+\frac{1}{2}-\frac{1}{8} \sqrt{\pi\left(1-z_{1}\right)}+O\left(\left(1-z_{1}\right)^{3 / 2}\right), \\
\beta z_{1} f_{2}^{\prime}\left(z_{1}\right)=\frac{\beta \sqrt{\pi}}{4}\left(1-z_{1}\right)^{-3 / 2}-\frac{3 \beta \sqrt{\pi}}{16}\left(1-z_{1}\right)^{-1 / 2}+O\left(\beta \sqrt{1-z_{1}}\right) .
\end{gathered}
$$

С учетом (22) отсюда следует, что $1-z_{1} \sim \frac{\beta}{2}$, и поэтому

$$
N\left(1-z_{1}\right)^{2} \sim \frac{n^{2}}{4 N}=o(1),
$$

так что

$$
z_{1}^{-N}=\exp \left(N\left(1-z_{1}\right)\right)(1+o(1))
$$

Полагая $1-z_{1}=\frac{\beta}{2}+\psi(\beta)$, имеем $\psi(\beta)=o(\beta)$ при $\beta \rightarrow 0$. Подставляя значение $1-z_{1}$ в приведенные выше оценки и используя (22), нетрудно вывести, что

$$
\psi(\beta)=-\frac{1}{\sqrt{2 \pi}}\left(\frac{1}{p}-\frac{1}{2}\right) \beta^{3 / 2}+o\left(\beta^{3 / 2}\right) .
$$

Далее получаем

$$
N\left(1-z_{1}\right)=\frac{N \beta}{2}+O\left(N \beta^{3 / 2}\right)=\frac{n}{2}+o(1), \text { если } n=o\left(N^{1 / 3}\right) .
$$

Таким образом,

$$
z_{1}^{-N}=e^{n / 2}(1+o(1))
$$

Кроме того,

$$
f_{2}\left(z_{1}\right) \sim \frac{\sqrt{\pi}}{2}\left(1-z_{1}\right)^{-1 / 2} \sim \sqrt{\frac{\pi}{2 \beta}}=\sqrt{\frac{\pi N}{2 n}},
$$




$$
\begin{gathered}
\left(1+p\left(f_{2}\left(z_{1}\right)-1\right)\right)^{n}=\exp \left(n \ln \left(p f_{2}\left(z_{1}\right)\right)+O\left(\frac{n}{f_{2}\left(z_{1}\right)}\right)\right)= \\
=\exp \left(n\left(\ln p+\ln f_{2}\left(z_{1}\right)\right)\right)\left(1+O\left(\sqrt{\frac{n^{3}}{N}}\right)\right)=p^{n} \exp \left(n \ln f_{2}\left(z_{1}\right)\right)(1+o(1)),
\end{gathered}
$$

и так как

$$
f_{2}\left(z_{1}\right)=\frac{\sqrt{\pi}}{2}\left(1-z_{1}\right)^{-1 / 2}(1+O(\sqrt{\beta})),
$$

то

$$
\begin{aligned}
&\left(1+p\left(f_{2}\left(z_{1}\right)-1\right)\right)^{n}=p^{n} \exp \left(n \ln \left(\frac{\sqrt{\pi}}{2}\left(1-z_{1}\right)^{-1 / 2}\right)+O(n \sqrt{\beta})\right)= \\
&=\left(\frac{p \sqrt{\pi}}{2}\right)^{n} \exp \left(-\frac{n}{2} \ln \left(\frac{\beta}{2}\right)+O(n \sqrt{\beta})\right)=\left(\frac{p \sqrt{\pi}}{2}\right)^{n}\left(\frac{2}{\beta}\right)^{n / 2}(1+o(1))= \\
&=\left(p \sqrt{\frac{\pi N}{2 n}}\right)^{n}(1+o(1)),
\end{aligned}
$$

поскольку

$$
\ln \left(1-z_{1}\right)=\ln \left(\frac{\beta}{2}\right)+O(\sqrt{\beta})
$$

Наконец,

$$
f_{2}^{\prime \prime}\left(z_{1}\right) \sim \frac{3 \sqrt{\pi}}{8}\left(1-z_{1}\right)^{-5 / 2} \sim 3 \sqrt{\frac{\pi}{2}} \beta^{-5 / 2},
$$

так что

$$
\frac{1}{\sqrt{2 \pi N\left(z_{1} f_{2}^{\prime \prime}\left(z_{1}\right)\left(f_{2}^{\prime}\left(z_{1}\right)\right)^{-1}+1-\beta^{-1}\right)}}=\frac{1}{2 N} \sqrt{\frac{n}{\pi}}(1+o(1)) .
$$


Из полученных оценок и (21) при $m=2$ получаем асимптотическую формулу для $\mathbf{E} \xi_{2}$, приведенную в формулировке теоремы 8. Так как

$$
\left(1-z_{1}\right)^{-1} \sim \frac{2}{\beta}=\frac{2 N}{n}
$$

то

$$
\mathbf{E} \eta_{2} \sim \frac{2 N}{n} \mathbf{E} \xi_{2}
$$

Теорема 8 доказана.

Доказательство теоремы 9. Для обоснования оценки (26) используем соотношение (25), полагая в нём согласно (24)

$$
P\left(\left(1+z^{-1}\right)\left(z+u^{-1}\right)\right)=\exp \left(\lambda\left(z+u^{-1}+z^{-1} u^{-1}\right)\right) .
$$

Если обозначить

$$
f(z, u)=u(1+z)(1+u z) \exp \left(\beta^{-1}\left(z+u^{-1}+z^{-1} u^{-1}\right)\right),
$$

то с учетом (9), (25) имеем

$$
\mathbf{E} \xi_{1}^{2}=\frac{1}{(2 \pi i)^{2}} \oint_{|z|=z^{*}>0} \oint_{|u|=u^{*}>0} \frac{(f(z, u))^{N}}{z^{N+1} u^{N+1}} d z d u .
$$

Для нахождения точек перевала подынтегральной функции в (36) составим систему уравнений

$$
\left\{\begin{array}{l}
z \frac{\partial f}{\partial z}(z, u)=f(z, u), \\
u \frac{\partial f}{\partial u}(z, u)=f(z, u) .
\end{array}\right.
$$

Подставляя значение $f(z, u)$ из (35) и вычисляя частные производные $\frac{\partial f}{\partial z}(z, u)$ и $\frac{\partial f}{\partial u}(z, u)$ после элементарных преобразований, получаем эквивалентную систему

$$
\left\{\begin{array}{l}
\left(u z^{2}-1\right)(1+u \cdot z)=0, \\
\beta z^{2} u^{3}=1
\end{array}\right.
$$

При условии $u>0 ; z>0$ решение этой системы имеет вид

$$
z=z^{*}=\sqrt[4]{\beta}>0, \quad u=u^{*}=\frac{1}{\sqrt{\beta}}>0 .
$$


Из (35), (37) выводим, что

$$
f\left(z^{*}, u^{*}\right)=\left(\beta^{-1 / 8}+\beta^{-3 / 8}\right)^{2} \exp \left(2 \beta^{-3 / 4}+\beta^{-1 / 2}\right) .
$$

Применяя метод перевала к оценке интеграла в (36), получаем

$$
\mathbf{E} \xi_{1}^{2}=\frac{\left(f\left(z^{*}, u^{*}\right)\right)^{N}}{2 \pi N\left(z^{*}\right)^{N}\left(u^{*}\right)^{N} \sqrt{\Delta}}\left(1+O\left(N^{-1}\right)\right),
$$

где

$$
\begin{aligned}
& \Delta=\operatorname{det}\left(\Delta_{i, j}\right)(1 \leq i ; j \leq 2), \\
& \Delta_{11}=\left.z \frac{\partial}{\partial z}\left(z \frac{\partial(\ln f)}{\partial z}\right)\right|_{\substack{z=z^{*} \\
u=u^{*}}}=\frac{2}{2+z^{*}+z^{*} u^{*}}+2 \beta^{-1} z^{*} \text {, } \\
& \Delta_{22}=\left.u \frac{\partial}{\partial u}\left(u \frac{\partial(\ln f)}{\partial u}\right)\right|_{\substack{z=*^{*} \\
u=u^{*}}}=\frac{z^{*} u^{*}}{\left(1+z * u^{*}\right)^{2}}+\frac{1+z^{*}}{\beta z^{*} u^{*}} \\
& \Delta_{12}=\Delta_{21}=\frac{z^{*} u^{*}}{\left(1+z^{*} u^{*}\right)^{2}}+\frac{u^{*}}{z^{*}} .
\end{aligned}
$$

Из этих соотношений с учетом (37) и равенства

$$
\Delta=\Delta_{11} \Delta_{22}-\Delta_{12} \Delta_{21}
$$

следует, что величина $\Delta$ определяется по формуле, приведенной в формулировке теоремы 9.

Оценка (26) является следствием соотношений (37)-(39). Теорема 9 доказана.

Доказательство теоремы 10 проводится аналогично. Чтобы обосновать оценку (27), используем (25), полагая

$$
P\left(\left(1+z^{-1}\right)\left(z+u^{-1}\right)\right)=\left(1+p\left(z+u^{-1}+z^{-1} u^{-1}\right)\right)^{\beta \cdot N} .
$$

Обозначая

$$
g(z, u)=u(1+z)(1+u z)\left(1+p\left(z+u^{-1}+z^{-1} u^{-1}\right)\right)^{\beta},
$$

из (20), (25) выводим, что

$$
\mathbf{E} \xi_{1}^{2}=\frac{1}{(2 \pi i)^{2}} \oint_{|z|=z^{*}>0 \mid} \oint_{|u|=u^{*}>0} \frac{(g(z, u))^{N}}{z^{N+1} u^{N+1}} d z d u .
$$


Решая аналогичную систему уравнений с заменой функции $f(z, u)$ на $g(z, u)$, получаем, что при условии $u>0 ; z>0$

$$
u z^{2}=1,
$$

причем $z=z^{*}$ - единственный положительный корень уравнения, приведенного в формулировке теоремы 10. Таким образом

$$
u=u^{*}=\left(z^{*}\right)^{-2}>0
$$

(в этих соотношениях величины $z^{*}, u^{*}$ имеют, вообще говоря, другие значения по сравнению с теоремой 9).

Кроме того,

$$
g\left(z^{*}, u^{*}\right)=\left(1+\frac{1}{z^{*}}\right)^{2}\left(1+p z^{*}\left(z^{*}+2\right)\right)^{\beta} .
$$

Вычисляя определитель $\Delta$ согласно приведенным выше формулам с заменой $f(z, u)$ на $g(z, u)$, получаем его выражение в формулировке теоремы 10. Поскольку при наших условиях соотношение (39) остается справедливым (с указанной заменой), с учетом оценок (40)-(42) отсюда следует (27). Теорема 10 доказана.

\section{Список литературы}

1. Караиуба А. А. Основы аналитической теории чисел. - М.: Наука, 1983.

2. Фрейман Г. А. Проблема Варинга с растущим числом слагаемых // Учен. зап. Елабужск. гос. пед. института. - 1958. - Вып. 3. - С. 105-119.

3. Постников А. Г. Введение в аналитическую теорию чисел. - М.: Наука, 1971.

4. Тимамев $A$. Н. Обобщенная схема размещения в задачах вероятностной комбинаторики. - М.: Издательский дом «Академия», 2011.

5. Колчин В. Ф. Случайные отображения. - М.: Наука, 1984.

6. Тимамев $A$. Н. Асимптотические разложения в вероятностной комбинаторике. - М.: ТВП, 2011.

7. Тимамев $A$. Н. Большие уклонения в вероятностной комбинаторике. М.: Издательский дом «Академия», 2011.

8. Чандрасекхаран К. Арифметические функции. - М.: Наука, 1975. 